Research Article

\title{
An Electrochemical Sensor Based on Gold Nanodendrite/Surfactant Modified Electrode for Bisphenol A Detection
}

\author{
Nguyen Thi Lien, ${ }^{1}$ Le Quoc Hung, ${ }^{2}$ Nguyen Tien Hoang, ${ }^{3}$ Vu Thi Thu, ${ }^{3}$ Dau Thi Ngoc Nga, ${ }^{3}$ \\ Pham Thi Hai Yen, ${ }^{2}$ Pham Hong Phong, ${ }^{2}$ and Vu Thi Thu Ha $\mathbb{1}^{2,3}$ \\ ${ }^{1}$ Department of Chemistry, Hanoi University of Science, 19 Le Thanh Tong, Hanoi, Vietnam \\ ${ }^{2}$ Institute of Chemistry, Vietnam Academy of Science and Technology, 18 Hoang Quoc Viet, Cau Giay, Hanoi, Vietnam \\ ${ }^{3}$ University of Science and Technology of Hanoi, Vietnam Academy of Science and Technology, 18 Hoang Quoc Viet, Cau Giay, \\ Hanoi, Vietnam
}

Correspondence should be addressed to Vu Thi Thu Ha; vuthithuha503@gmail.com

Received 12 October 2020; Revised 30 November 2020; Accepted 14 December 2020; Published 29 December 2020

Academic Editor: Tien Duc Pham

Copyright (c) 2020 Nguyen Thi Lien et al. This is an open access article distributed under the Creative Commons Attribution License, which permits unrestricted use, distribution, and reproduction in any medium, provided the original work is properly cited.

\begin{abstract}
In the present work, we reported the simple way to fabricate an electrochemical sensing platform to detect Bisphenol A (BPA) using galvanostatic deposition of $\mathrm{Au}$ on a glassy carbon electrode covered by cetyltrimethylammonium bromide (CTAB). This material (CTAB) enhances the sensitivity of electrochemical sensors with respect to the detection of BPA. The electrochemical response of the modified GCE to BPA was investigated by cyclic voltammetry and differential pulse voltammetry. The results displayed a low detection limit $(22 \mathrm{~nm})$ and a linear range from 0.025 to $10 \mu \mathrm{m}$ along side with high reproducibility $(\mathrm{RSD}=4.9 \%$ for seven independent sensors). Importantly, the prepared sensors were selective enough against interferences with other pollutants in the same electrochemical window. Notably, the presented sensors have already proven their ability in detecting BPA in real plastic water drinking bottle samples with high accuracy (recovery range $=96.60 \%-102.82 \%$ ) and it is in good agreement with fluorescence measurements.
\end{abstract}

\section{Introduction}

Bisphenol A (BPA) is known as a typical endocrine disruptor in the environment, which is widely used in the production of plastic products [1]. As a result, BPA is found in food and drinking water from product packaging, and therefore humans may routinely ingest amounts of BPA even in trace levels [2]. So far, different analytical methods have been developed and used for the determination of BPA, such as gas chromatography [3], high performance liquid chromatography [4], fluorimetry [5], molecular imprinting [6], and enzyme-linked immunosorbent assay [7]. Electrochemical detection techniques have advantages in relation to these techniques of rapidity, low cost, high sensitivity, simple operation, good selectivity, and real-time detection with in situ analysis [8]. Recently, electrochemical techniques have been popularly developed for BPA detection [9-28], especially many publications focus on electrode modification in order to enhance signals of target analytes. Recently, lots of different modifiers have been developed for electrochemical sensors such as carbon black/f-MWCNT composite modified glassy carbon electrode (GCE) which was used for detection of BPA with a detection limit of $0.08 \mu \mathrm{mol} \mathrm{L}^{-1}$ [9], molybdenum disulfide nanoflower-chitosan- $\mathrm{Au}$ nanoparticle composites [10], poly(amidoamine)-AuNP-silk fibroin [29], carbon nanotubes- $\beta$-cyclodextrin [30], and carboxylic group functionalized CNT-poly(3, 4-ethylenedioxythiophene) [31]. Besides that, gold nanoparticles (AuNP) combined with other materials were of interest to many researchers, such as multiwall carbon nanotubes 
(MWCNT) plus AuNP modified GCE [11, 32, 33] because $\mathrm{Au}$ is the material with extremely high conductivity. As Najib Ben Messaoud et al. [11] showed, a modified electrode was prepared by two steps: drop-casting a desired amount of MWCNT on polished GCE followed by deposition of AuNP on the surface of the previously prepared MWCNT/GCE. With this sensor, the detection limit of BPA reached $4 \mathrm{nM}$. In this work, an electrochemical sensor for BPA detection consisting of gold nanodendrites (AuNDs) deposited on GCE, which was precovered by cetyltrimethylammonium bromide (CTAB). CTAB is one of the known cationic surfactant which has been extensively employed to enhance the sensitivity of electrochemical sensors [34, 35]. It is also presented that CTAB has a hydrophilic head on one side and a long hydrophobic tail on the other side of its molecule. Also, as reported, surfactants adsorbed at the electrode surface may alter the electrode behavior and accelerate the electron transfer process between the analyte and the electrode surface [36]. In this context, the GCE-supported $\mathrm{Au}$ nanodendrites and CTAB (denoted as AuNDs/CTAB/ GCE) were demonstrated as an electrochemical sensor, which has sensitive electrochemically active surface areas due to the hierarchical nanodendrites structure and the formation of nanodendrite on the CTAB/GCE was accomplished in just 5 minutes by simple one-step electrodeposition. The electrochemical behavior of BPA on this sensor was studied by cyclic voltammetry $(\mathrm{CV})$ and electrochemical impedance spectroscopy (EIS), and the analytical measurements were performed by differential pulse voltammetry (DPV). The AuNDs/CTAB/GCE sensor has been employed to detect BPA in aqueous samples and important factors related to electrochemical response, such as $\mathrm{pH}$ (from 5.0 to 9.0), were examined as well to get the optimal conditions for BPA detection.

\section{Materials and Methods}

2.1. Materials and Sensor Preparation. Bisphenol A (BPA) was purchased from Sigma-Aldrich (Structures in Figure S1). GCE (BAS, diameter $=3 \mathrm{~mm}$ ) was used as a current collector. Phosphate buffer solution (PBS) was prepared by mixing $0.2 \mathrm{M} \mathrm{KH}_{2} \mathrm{PO}_{4}$ and $0.2 \mathrm{MK}_{2} \mathrm{HPO}_{4}$ until the desired $\mathrm{pH}$ (in the range from 5.0 to 9.0) was obtained. Potassium ferrocyanide trihydrate $\left(\mathrm{K}_{4}\left[\mathrm{Fe}(\mathrm{CN})_{6}\right] .3 \mathrm{H}_{2} \mathrm{O}\right)$, potassium ferricyanide $\left(\mathrm{K}_{3}\left[\mathrm{Fe}(\mathrm{CN})_{6}\right]\right)$, and all reagents needed for the construction of $\mathrm{Au}$ nanodendrites, $\mathrm{HAuCl}_{4}$ (99.999\%), KI, $\mathrm{H}_{2} \mathrm{SO}_{4}$, and $\mathrm{NH}_{4} \mathrm{Cl}$ were bought from SigmaAldrich. All chemicals were used as received without any further treatments.

The AuNDs/CTAB/GCE sensor was prepared as reported in our previous publication $[37,38]$. In brief, $5 \mu \mathrm{L}$ $\mathrm{CTAB}$ was drop-casted on GCE, dried in the atmosphere in about $2 \mathrm{~h} 30$ to $3 \mathrm{~h}$. The dried CTAB/GCE was put in a solution containing $20 \mathrm{mM} \mathrm{HAuCl}_{4}, 1 \mathrm{mM} \mathrm{KI}, 5 \mathrm{M} \mathrm{NH} \mathrm{NCl}_{4}$, and $0.5 \mathrm{M} \mathrm{H}_{2} \mathrm{SO}_{4}$, and previous regime published by author's group was followed [37, 38]. A current of $-20 \mathrm{~mA}$ was applied to it for the preparation of $\mathrm{Au}$ nanodendrites by electrodeposition for $30 \mathrm{~s}$. AuNDs/CTAB/GCE was rinsed through by a mixture of EtOH and $\mathrm{H}_{2} \mathrm{O}(1: 3)$ and kept in a desiccator overnight. A scanning electron microscope (SEM) image of the built Au nanodendrite was obtained (S-4800, Hitachi, Japan) with an electron beam accelerated by a voltage of $15-20 \mathrm{kV}$ to characterize the structure. Fluorescence spectra were obtained with a FluoroMax- 4 spectrofluorometer equipped with an Ozone-free xenon arc lamp (150 W), Czerny-Turner monochromators (HORIBA-Japan). All measurements were performed in a $10 \mathrm{~mm}$ quartz cell at room temperature. The emission spectra are recorded between 470 and $540 \mathrm{~nm}$ with the excitation wavelength at $460 \mathrm{~nm}$.

2.2. Electrochemical Measurements of Samples. All of the electrochemical measurements were conducted at room temperature $\left(25^{\circ} \mathrm{C} \pm 1\right)$ using a three-electrode system, in which an $\mathrm{Ag} / \mathrm{AgCl}$ electrode and a Pt wire were used as the reference and auxiliary electrode, respectively. A custommade, multifunctional potentiostat-galvanostat manufactured by this research group (Vietnam Academy of Science and Technology, Hanoi, Vietnam) was used. It was equipped with 12-byte analog-digital and digital-analog converters with two operational amplifiers, and it provided the current resolution down to $0.008 \mathrm{nA}$.

DPV was used for BPA measurement with a differential step of $0.005 \mathrm{~V}$, a sampling time of $0.04 \mathrm{~s}$, a pulse width of $0.08 \mathrm{~s}$, and a pulse amplitude of $0.05 \mathrm{~V}$. The accumulation conditions of BPA onto AuNDs/CTAB/GCE were conducted at $i=0$ (under open-circuit potential) with a duration time of $240 \mathrm{~s}$ (selected from previous publications [39] and voltammograms were recorded over a range of 0.25 to $0.85 \mathrm{~V}$ versus $\mathrm{Ag} / \mathrm{AgCl}$. A PBS solution ( $\mathrm{pH} 7$ ) was used as a supporting electrolyte in all measurements.

\section{Results and Discussion}

3.1. Surface Morphology of Prepared Sensors. The surface morphology of AuNDs/CTAB/GCE was examined using the SEM technique (Figure 1). Au nanodendrites were quickly formed on the electrode surface after 5-minutes of electrodeposition. When CTAB surfactant was not used, the aggregation of gold nanostructures (Figure S2) and the formation of large gold branches (Figure 1(c)) were observed. The use of CTAB has helped to limit those unwanted defects, thus significantly increased the active surface area and provided more access sites for analytes at not only the tip of gold brands but also inside gold branches.

\subsection{Electrochemical Performance of AuND/CTAB/GCE.} Before using AuNDs/CTAB/GCE prepared for detection of BPA on GCE, CTAB/GCE, AuNDs/GCE, and AuNDs/ CTAB/GCE, electrochemical impedance spectroscopy (EIS) was recorded in $0.2 \mathrm{M}$ PBS containing $5 \mathrm{mM} \mathrm{Fe}(\mathrm{CN})_{6}{ }^{3-/ 4-}$. In the Nyquist diagram (Figure 2), the bare GCE exhibited a relatively low electron transfer resistance $\left(R_{\mathrm{ct}}=146.3 \Omega\right)$ with a small semicircle (refer to in inset for further detail). After the drop-casting CTAB and deposition of AuNDs, the electron transfer rate dramatically increased, which resulted from a decrease of the charge transfer resistance $\left(R_{c t}=25.4 \mathrm{O}\right.$ 


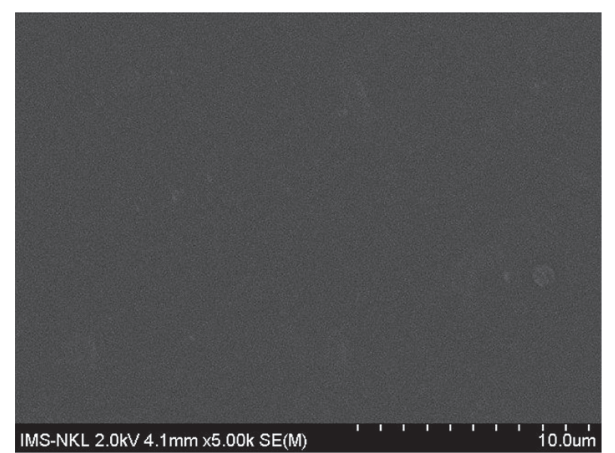

(a)

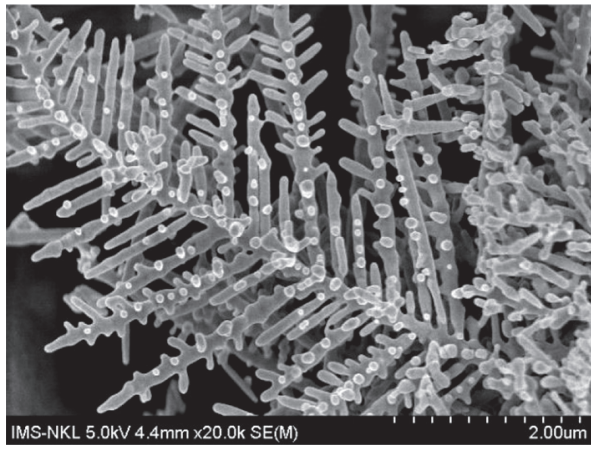

(c)

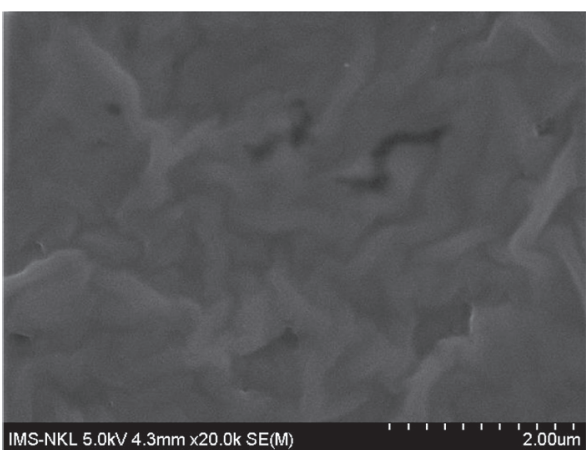

(b)

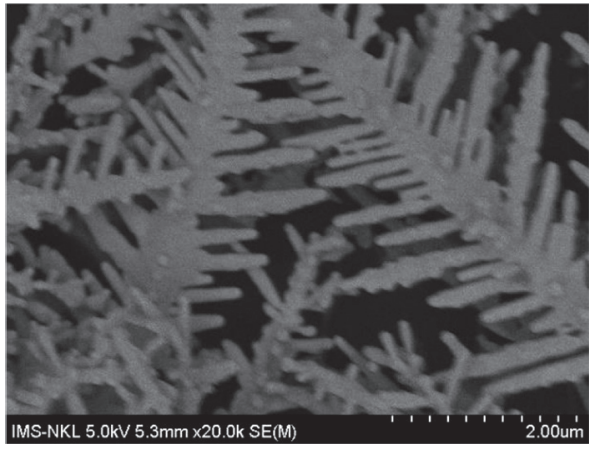

(d)

Figure 1: SEM images of (a) GCE, (b) CTAB/GCE, (c) AuNDs/GCE, and (d) AuNDs/CTAB/GCE samples.

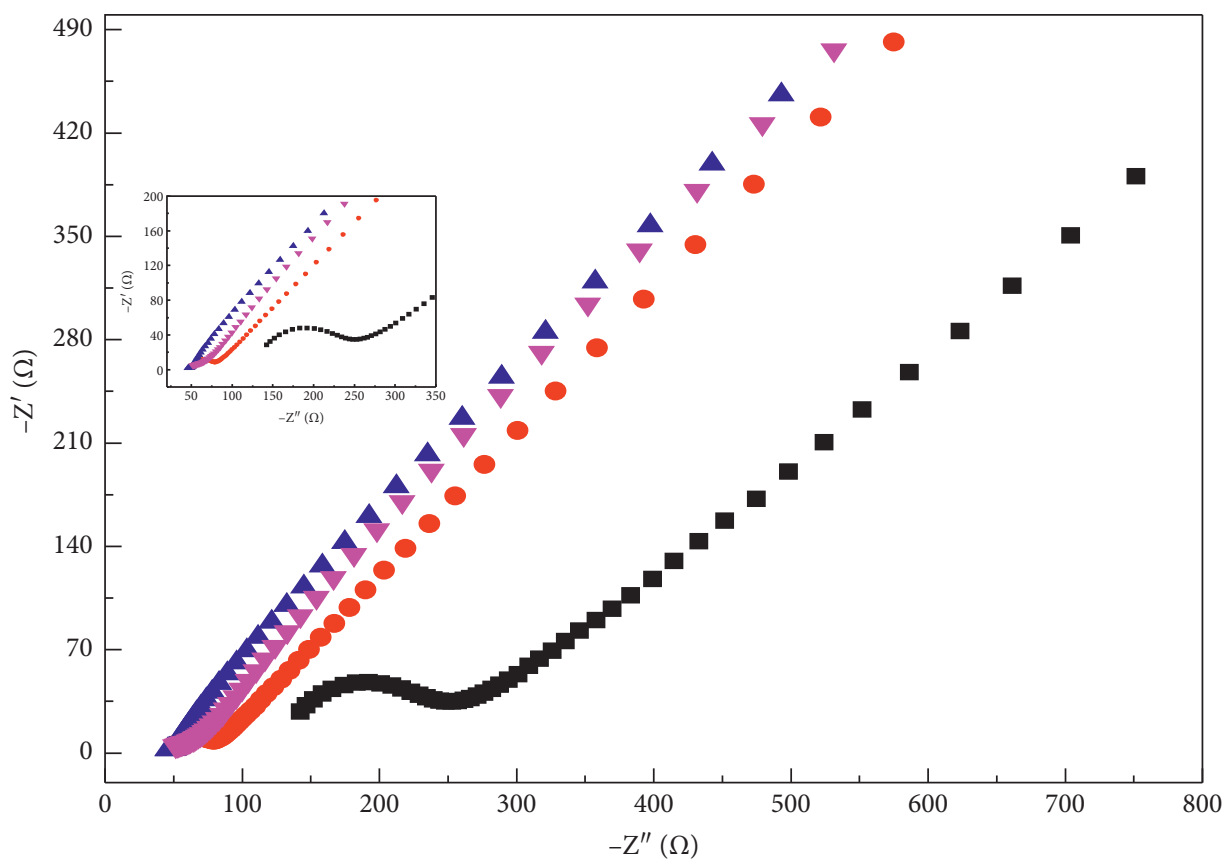

- GCE

- CTAB/GCE

- AuNDs/GCE

- AuNDs/CTAB/GCE

Figure 2: Nyquist diagrams of GCE, CTAB/GCE, AuNDs/GCE, and AuNDs/CTAB/GCE in the solution containing 5 mM Fe(CN) ${ }_{6}^{3-/ 4-}$ and $0.1 \mathrm{M}$ PBS. Parameters are as follows: Frequency range from $0.1 \mathrm{~Hz}$ to $10000 \mathrm{~Hz}$, initiative potential: $0.23 \mathrm{~V}$, amplitude: $10 \mathrm{mV}$, and quiet time of $5 \mathrm{~s}$. 
only) due to the high conductivity of AuNDs on the electrode surface. Also, CTAB molecules must have aided to improve the electron transfer process of $\mathrm{Fe}(\mathrm{CN})_{6}{ }^{3-/ 4-}$ probe at electrode surface [35]. All the results further demonstrate that the electrode is well done with the presence of AuNDs layers on its surface. This result is in good agreement with $\mathrm{CV}$ tests (Figure 3 ).

In Figure 3, it is seen that in a well-defined reversible redox system with a peak separation $\left(\Delta \mathrm{E}_{\mathrm{p}}\right)$ of $\sim 80 \mathrm{mV}$ for all cases, the CTAB/GCE exhibits a pretty low electron transfer rate. After deposition of AuNDs, the oxidation peak of this redox probe increased almost three times higher than $\mathrm{CTAB} / \mathrm{GCE}$ only in terms of electrical conductivity (Figure 3). Also, as stated in [36], CTAB can help in accelerating the electron transfer process between the analyte and the electrode surface. Therefore the total peak current received on AuNDs/CTAB/GCE was the highest.

3.3. Effects of Scan Rate (v). Kinetics of electrochemical oxidation of BPA on AuNDs/CTAB/GCE was investigated in PBS at pH 7 containing $10 \mu \mathrm{m}$ target analyte with different scan rates from 10, 20, 50, 100, and $200 \mathrm{mV} \mathrm{s}^{-1}$ (Figure 4). Oxidation peak currents appeared at $0.55 \mathrm{~V}$ and increased linearly with the increasing scan rate as follows: $i_{p, a}=0.0316 \times v+0.7415\left(R^{2}=0.9993\right)$, which suggests an adsorption controlled kinetic process on the modified electrode surface AuNDs/CTAB/GCE [19-28]. As seen in Figure 4 , the oxidation peak potential $\left(E_{p, a}\right)$ slightly shifted to more positive potentials with the increase of the scan rate. The dependence between $E_{p, a}$ and the $\ln (v)$ was given: $E_{p r a}(\mathrm{~V})=0.0184 \times \ln (v)+0.4949 \quad\left(R^{2}=0.9969\right) \quad$ (inset of Figure 4(b)), and the number of electrons transferred estimated from Laviron quation is as follows [40]:

$$
E_{p, a}=E^{o}+\left(\frac{\mathrm{RT}}{\alpha n F}\right) \ln \left(\frac{\mathrm{RT} k^{o}}{\alpha n F}\right)+\left(\frac{\mathrm{RT}}{\alpha n F}\right) \ln (v)
$$

where $v$ is the scan rate, $n$ is the number of electrons transferred, $\alpha$ is the electron transfer coefficient, $\mathrm{k}^{\mathrm{o}}$ is the standard rate constant of the reaction, and $R, \mathrm{~F}$, and $T$ are gas constant, Faraday constant, and absolute temperature, respectively. As the slope of the plot of $E_{p, a}$ versus $\ln (v)$ (equal to $\left.\mathrm{RT} / \alpha_{\mathrm{n}} \mathrm{F}\right)$ is 0.0184 , the value of $\alpha_{n}$ was about 1.3. Also, from Laviron [40], $\alpha$ for an irreversible electrode process is assumed to be 0.5. Therefore, the number of electrons transferred $(n)$ for electrooxidation of BPA is around 2 [26-28].

3.4. Effect of $p H$. It is known that the electrochemical oxidation of BPA followed a proton coupled electron transfer mechanism, i.e., $\mathrm{BPA} \rightleftharpoons$ oxidized product $+\mathrm{ne}^{-}+\mathrm{nH}^{+}(n=1$ or 2); the reaction rate is directly depending on the electron flux and the concentration of proton in solution. Consequently, the $\mathrm{pH}$ becomes one of the key parameters impacting the performance of the presented sensors. DPVs of BPA $(C=50 \mu \mathrm{m})$ were recorded by sweeping the potential from $0.1 \mathrm{~V}-0.9 \mathrm{~V}$ in $\mathrm{PBS}$ buffer with $\mathrm{pH}$ ranging from 5.0-9.0 at interval 1.0. It was found that the peak potential is

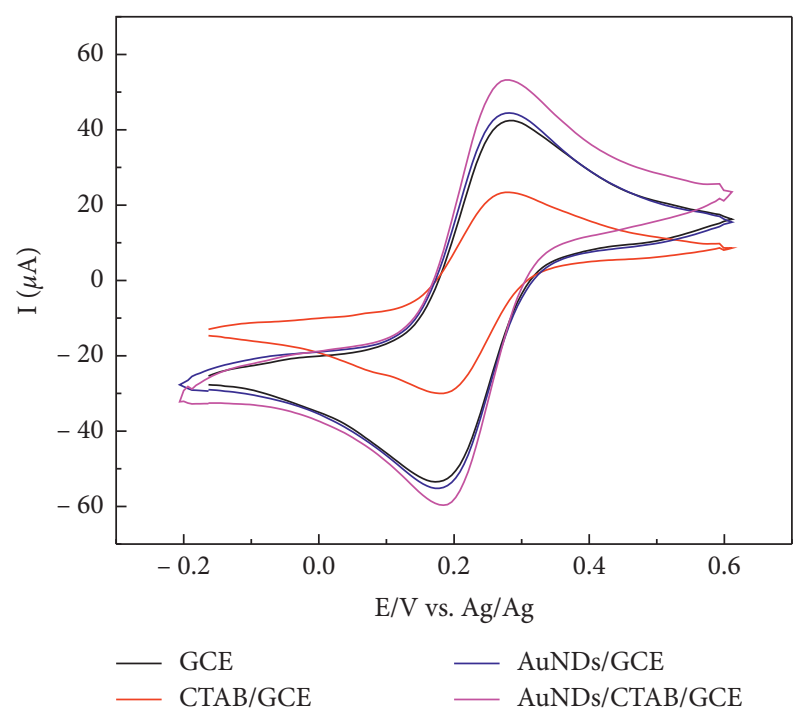

Figure 3: CVs of GCE, CTAB/GCE, AuNDs/GCE, and AuNDs/ $\mathrm{CTAB} / \mathrm{GCE}$ in $5 \mathrm{mM} \mathrm{Fe}(\mathrm{CN})_{6}{ }^{3-}$ and $0.2 \mathrm{M} \mathrm{PBS}$, from -0.2 to $0.6 \mathrm{~V}$ versus $\mathrm{Ag} / \mathrm{AgCl}$.

shifted to a more negative direction at higher $\mathrm{pH}$ values (Figure 5(a)).

By plotting the variation of peak potential depending on $\mathrm{pH}$ values, a linear relationship is obtained, i.e., $E_{p, a}=-0.0659$ $\mathrm{pH}+0.9642, R^{2}=0.9869$. A slope of $0.065 \mathrm{~V} / \mathrm{pH}$ was pretty close to the theoretical value of $0.059 \mathrm{~V} / \mathrm{pH}$, showing that the transfer of electrons was accompanied by an equal number of protons in electrode reaction. According to the calculation in [41] $-0.065 x / n=-0.059$, where $n$ is the transferred electron number and $x$ is the number of protons involved in the reaction, as mentioned above, the number of electrons transferred is about 2, the electrochemical oxidation of BPA is a two-electron and two-proton process, which is in good agreement with previous publications [26-28] as below.

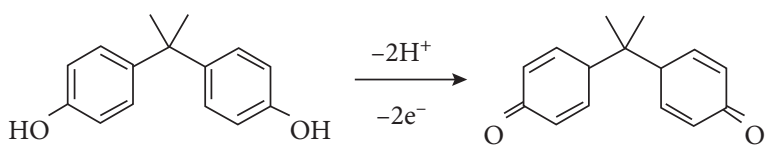

3.5. Detection of BPA Using AuNDs/CTAB/GCE. In order to compare electrochemical signals BPA on four materials used for the sensor, GCE, CTAB/GCE, AuNDs/GCE, and AuNDs/ CTAB/GCE, DPV signals were recorded in PBS pH 7. Figure 6 describes peak currents of BPA on four above materials at $1.0,5.0$, and $10 \mu \mathrm{m}$. It is seen that currents measured on AuNDs/CTAB/GCE are always highest and it is almost 10-, 4, and 2-fold compared with it on GCE, CTAB/GCE, AuNDs/ GCE, respectively. Also, it was observed visually that with AuNDs/GCE only, AuNDs layers easily sloughed from the GCE surface. By measuring CVs of AuNDs/CTAB/GCE at different scan rates in $0.2 \mathrm{M} \mathrm{PBS}$ containing $5 \mathrm{mM} \mathrm{Fe}(\mathrm{CN})_{6}{ }^{3-1}$ ${ }^{4-}$, electrochemical active surface of this sensor is not a significant difference in comparison with bare GCE (Figure S3). Therefore, it is assumed that the increase of peak current was due to the fact that AuNDs might provide more 


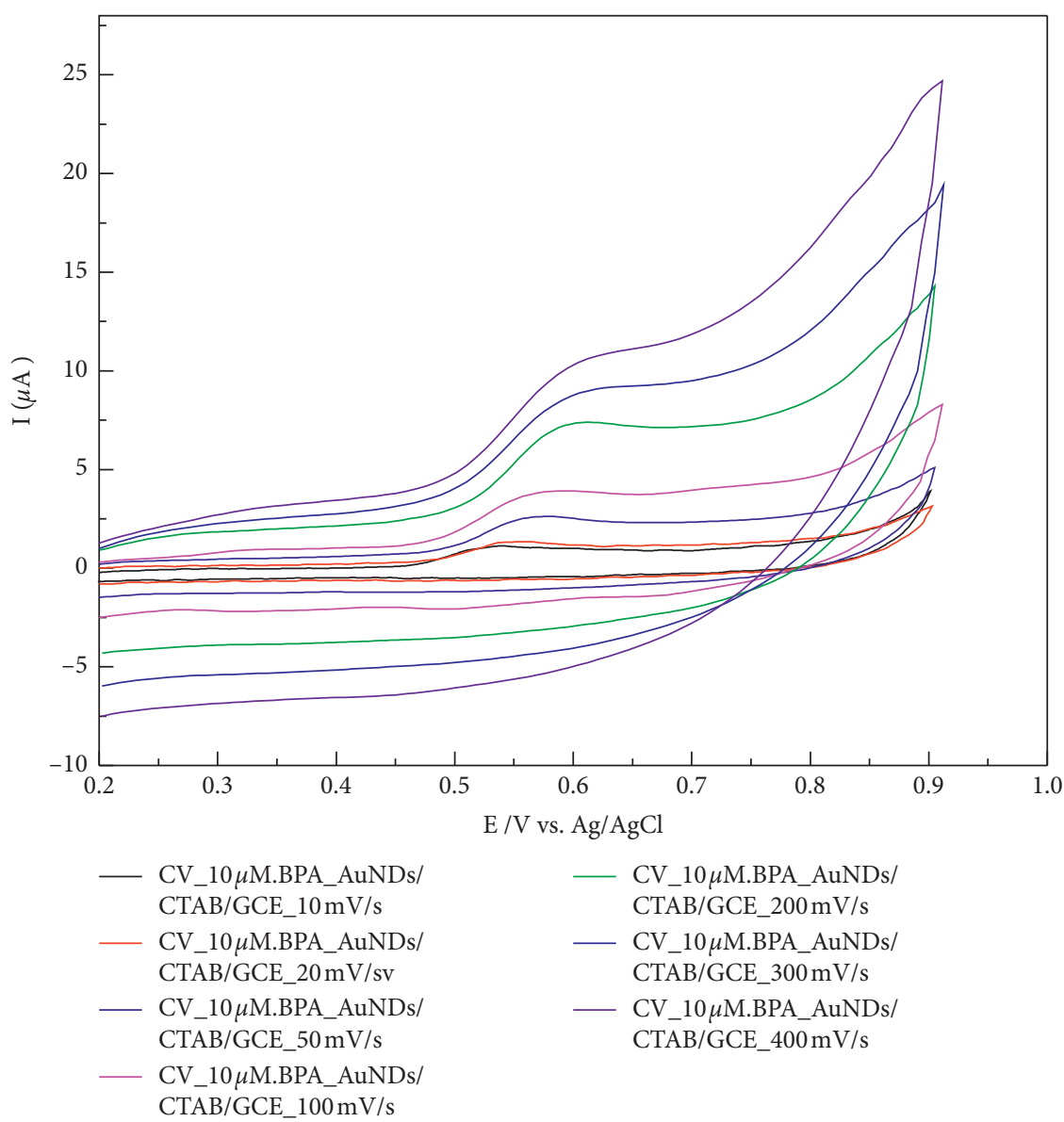

(a)

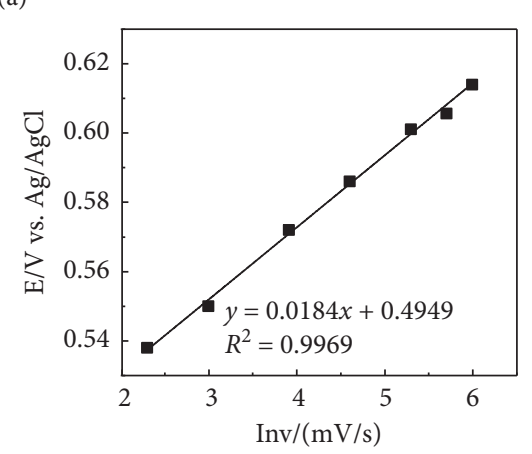

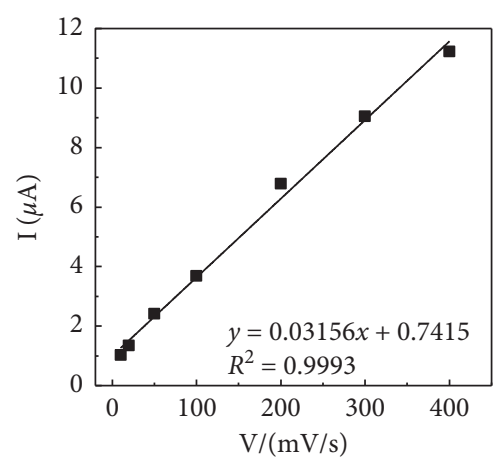

(c)

Figure 4: (a) CVs of BPA $10 \mu \mathrm{m}$ in PBS pH 7 recorded on AuNDs/CTAB/GCE at different scan rates $(\nu): 10,20,50,100$, and $200 \mathrm{mVs}{ }^{-1}$. (Inset) Relationship (b) between anodic peak currents and scan rate and (c) between potentials and $\ln (\nu)$.

electroactive sites for BPA electrooxidation, while $\mathrm{CTAB}$ could improve adherence of targeted molecules and promote electron transfer process at electrode surface [35].

3.6. Calibration Curve. Figure 7(a) shows voltammograms of all the BPA samples measured using selected AuNDs/ CTAB/GCE under optimized conditions. BPA peak heights increased with an increase in concentrations with the regression equation as follows: $i(\mu \mathrm{A})=0.0012 \times C_{\mathrm{BPA}}(\mu \mathrm{m})+$ $0.007\left(R^{2}=0.9996\right)($ Figure $7(\mathrm{~b}))$.
The limit of detection of the sensor (LOD) was estimated $(\mathrm{LOD}=3.3 \times S D / b$; $S D$ : standard deviation of ordinate intercept, $b$ : slope of regression line) to be $22 \mathrm{~nm}$ for BPA in PBS (pH 7). It showed that the LOD of AuNDs/ CTAB/GCE sensor was comparable with those of other electrode-based sensors (Table 1) and much lower than the allowable level of BPA declared by WHO [42]. The excellent performance of AuNDs/CTAB/GCE based sensor for BPA benefits from the high conductivity, large area of AuNDs, and charge transfer promotion along long alkane chain of CTAB [35]. 


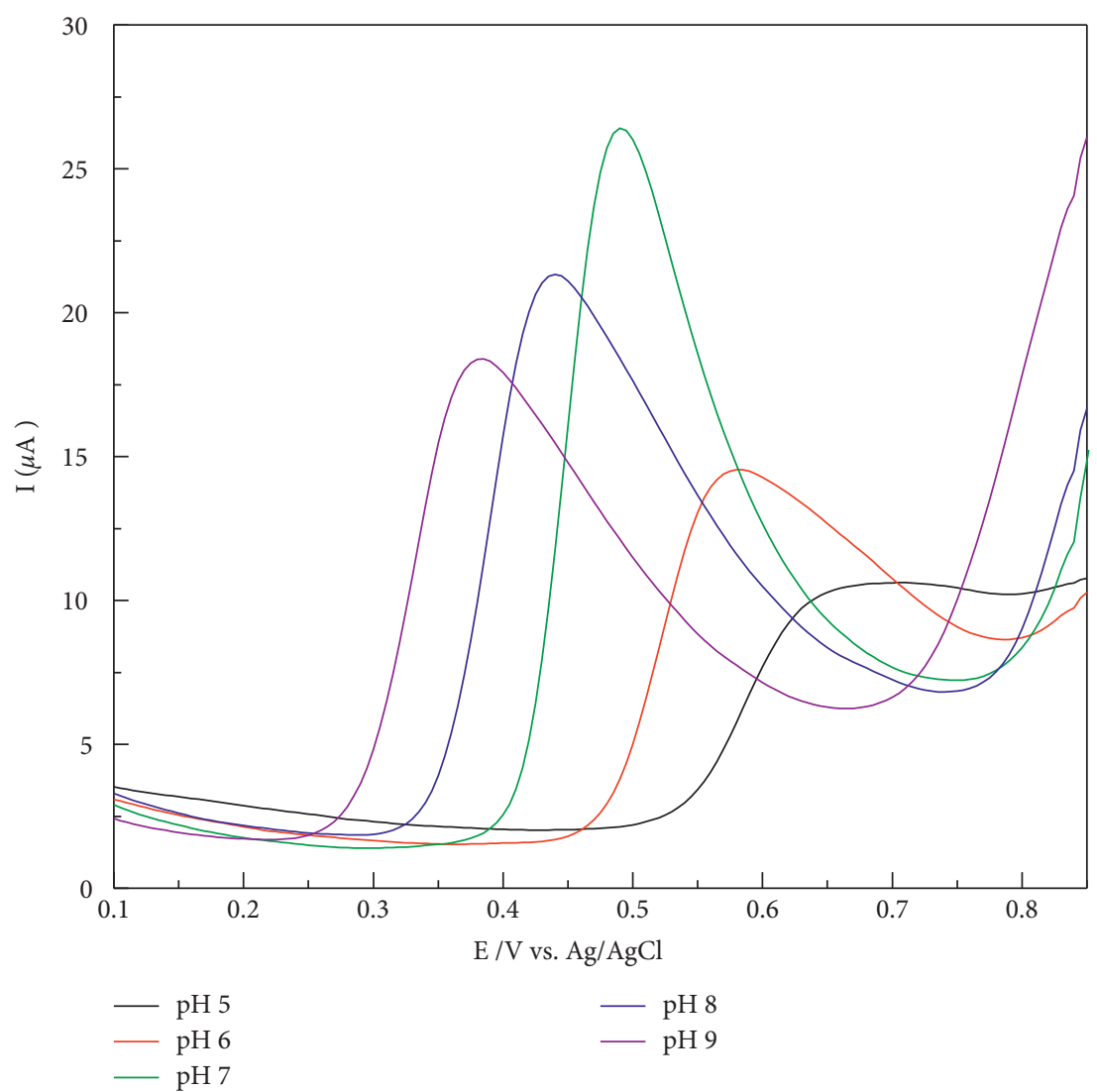

(a)

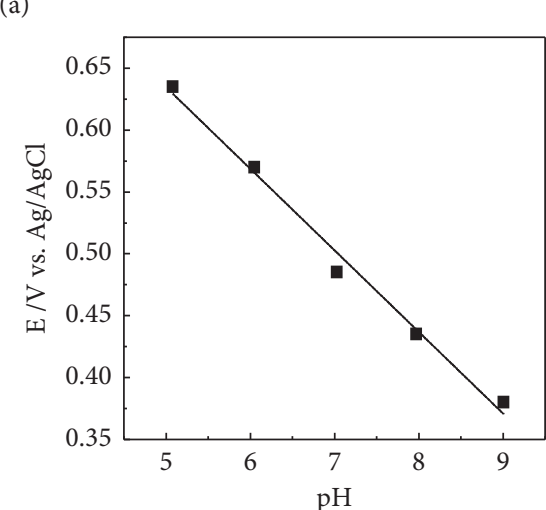

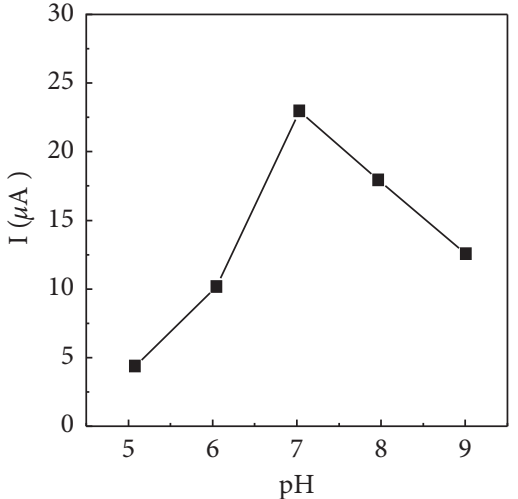

(b)

(c)

Figure 5: DPVs of $50 \mu \mathrm{m}$ BPA on AuNDs/CTAB/GCE in PBS pH 7 with different pH: 5.0; 6.0; 7.0; 8.0, and 9.0 (a). Currents varied by pH (b) and relationship between potentials and $\mathrm{pH}(\mathrm{c})$.

3.7. Reproducibility, Repeatability, and Interference Study. The reproducibility was tested using seven separated sensors (Figure S4). The magnitudes of error bars are quite small and the average relative standard deviations (RSDs) in the measurements of BPA were less than 5.0\%, thereby confirming the reproducible formation of AuNDs/CTAB on GCE. Repeatability tests have been done with 5 consecutive measurements using one AuNDs/CTAB/GCE sensor. RSDs received for $10 \mu \mathrm{m} \mathrm{BPA}$ in solutions was $4.37 \%$, less than $5.0 \%$ as above.

Finally, the selectivity of as-prepared sensors was evaluated in the presence of $\mathrm{Cu}^{2+} / \mathrm{Pb}^{2+} / \mathrm{Cd}^{2+}$ and 4-nitrophenol as interferants. The concentrations of interferants were designedly fixed at $100 \mu \mathrm{m}$, which is 20 times greater than BPA concentration. No significant changes in current intensity observed, suggesting no interference effect due to other compounds in this study (Figure S5).

3.8. Real Sample Analysis. To open the application, AuNDs/ CTAB/GCE was further used to determine BPA quantities in real samples prepared from a plastic drinking water bottle. Sample preparation from plastic pieces was treated as in [43]. Briefly, the plastic sample of LaVie bottles, a popular 


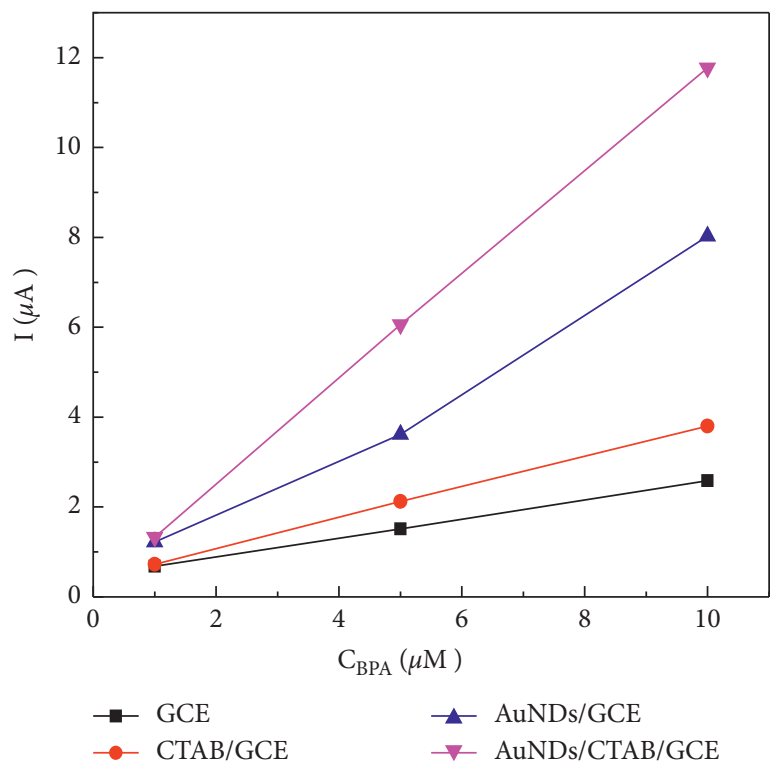

Figure 6: Peak current of BPA at 1.0, 5.0, and $10.0 \mu \mathrm{m}$ on GCE, CTAB/GCE, AuNDs/GCE, and AuNDs/CTAB/GCE recorded in PBS pH 7.

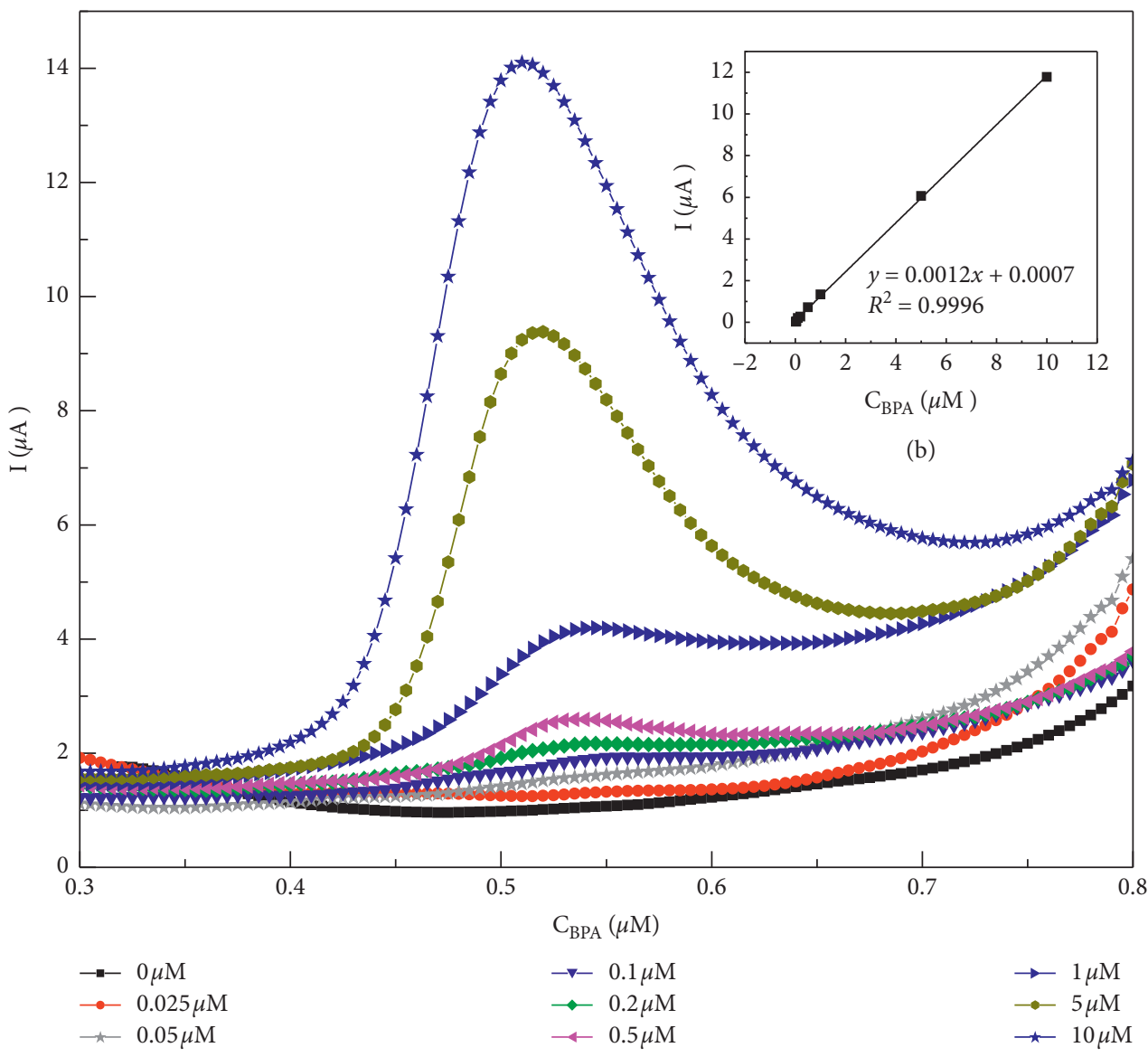

(a)

Figure 7: (a) Voltammograms recorded from BPA in the concentration range from 0.025 to $10 \mu \mathrm{m}$ using AuNDs/CTAB/GCE and (b) the variations of peak intensities according to the concentration change. 
TABLE 1: Comparison of sensing performances of electrochemical sensors for detection of BPA.

\begin{tabular}{|c|c|c|c|c|}
\hline Electrode configuration & Method & Linear range $(\mu \mathrm{m})$ & LOD $(\mu \mathrm{m})$ & Ref. \\
\hline CB/f-MWCNTs & $\mathrm{CV}$ & $0.1-130$ & 0.08 & [9] \\
\hline $\mathrm{AuNPs} / \mathrm{MoS}_{2} / \mathrm{GCE}$ & $\mathrm{CV}$ & $0.05-100$ & $5 \times 10^{-3}$ & {$[10]$} \\
\hline SWCNTs/CD/GCE & DPV & $0.0108-18.5$ & $1 \times 10^{-3}$ & {$[30]$} \\
\hline NPs/MWCNT/GCE & & $0.01-0.7$ & $4 \times 10^{-3}$ & {$[11]$} \\
\hline rGO-NiS & DPV & $4.38 \times 10^{-3}-0.438$ & $1.75 \times 10^{-3}$ & {$[12]$} \\
\hline $\mathrm{Cu}-\mathrm{BTC} / \mathrm{GCE}$ & DPV & $5 \times 10^{-3}-2$ & $0.72 \times 10^{-3}$ & {$[13]$} \\
\hline $\mathrm{Fe}_{3} \mathrm{O}_{4} \mathrm{NPs}-\mathrm{CB}$ & DPV & $0.1 \times 10^{-3}-50$ & $0.031 \times 10^{-3}$ & {$[14]$} \\
\hline RGO-Ag/GCE & DPV & $1-80$ & 0.54 & {$[15]$} \\
\hline $\mathrm{Rh}_{2} \mathrm{O}_{3}-\mathrm{GO} / \mathrm{GCE}$ & $\mathrm{CV}$ & $0.6-40$ & 0.12 & {$[16]$} \\
\hline $\mathrm{Cu}-\mathrm{Zn} / \mathrm{GO} / \mathrm{GCE}$ & SWV & $3 \times 10^{-3}-100$ and $350-20000$ & $0.88 \times 10^{-3}$ & {$[17]$} \\
\hline GO-CNTs- $\mathrm{Fe}_{3} \mathrm{O}_{4}$ & DPV & $3 \times 10^{-3}-0.2$ and $0.2-30.0$ & $1 \times 10^{-3}$ & {$[18]$} \\
\hline CTAB/MWCNTs/PGE & SWV & $2 \times 10^{-3}-0.808$ & $0.134 \times 10^{-3}$ & {$[19]$} \\
\hline $\mathrm{CS} / \mathrm{N}-\mathrm{GS} / \mathrm{GCE}$ & $\mathrm{CV}$ & $0.01-1.3$ & $5 \times 10^{-3}$ & {$[20]$} \\
\hline $\mathrm{BmimPF}_{6} / \mathrm{GN} / \mathrm{GCE}$ & LSV & $0.02-2000$ & $8 \times 10^{-3}$ & {$[21]$} \\
\hline GA/MWCNT- $\mathrm{NH}_{2}$ & DPV & $0.1-10$ & 0.02 & {$[22]$} \\
\hline $\mathrm{Cu}_{2} \mathrm{O}-\mathrm{rGO}$ & DPV & $0.1-80$ & 0.053 & [23] \\
\hline AuPdNPs/GNs & DPV & $0.05-10$ & $8 \times 10^{-3}$ & {$[24]$} \\
\hline PCE/PEDOT/BMIMBr & DPV & $0.1-500$ & 0.02 & [25] \\
\hline MoS2-SPAN/GCE & DPV & $0.001-1.0$ & $0.6 \times 10^{-3}$ & {$[26]$} \\
\hline ELDHs/GCE & DPV & $0.02-1.51$ & $6.8 \times 10^{-3}$ & [27] \\
\hline $\mathrm{CTAB} / \mathrm{Ce}-\mathrm{MOF} / \mathrm{GCE}$ & DPV & $0.005-50$ & $2 \times 10^{-3}$ & {$[28]$} \\
\hline AuNDs/CTAB/GCE & DPV & $0.025-10$ & $22 \mathrm{nM}$ & This work \\
\hline
\end{tabular}

AuNPs $=$ gold nanoparticles, $\mathrm{CPE}=$ carbon paste electrode, $\mathrm{NPs}=$ nanoparticles, ILs $:$ ionic liquids, $\mathrm{BTC}=$ benzenetricarboxylic, GO $=$ graphene oxide, $\mathrm{ErGO}=$ electrochemical reduced graphene oxide, GCE = glassy carbon electrode; SWV = square wave voltammetry, DPV = differential pulse voltammetry, $\mathrm{CV}=$ cyclic voltammetry, LSV = linear sweep voltammetry, MWNT = multiwalled carbon nanotubes, $\mathrm{GN}=$ graphene, CTAB = cetyltrimethylammonium bromide, $\mathrm{MOF}=$ metal organic frames, $\mathrm{PGE}=$ pencil graphite electrode, $\mathrm{CS}=$ chitosan, $\mathrm{GS}=$ graphene sheet, $\mathrm{BmimPF}_{6}=-\mathrm{butyl}-3-$ methylimidazoliumhexafluorophosphate.

TABLE 2: Determined BPA concentrations spiked in the real sample using AuNDs/CTAB/GCE and by Fluorescence methods.

\begin{tabular}{|c|c|c|c|c|c|}
\hline \multirow{3}{*}{ Sample } & \multicolumn{5}{|c|}{$\mathrm{BPA}(\mu \mathrm{m})$} \\
\hline & \multirow[b]{2}{*}{ Added } & \multicolumn{2}{|l|}{ Found } & \multicolumn{2}{|c|}{ Recovery (\%) } \\
\hline & & $\begin{array}{l}\text { By AuNDs/CTAB/GCE } \\
\text { sensor }\end{array}$ & By fluorescence & $\begin{array}{l}\text { By AuNDs/CTAB/GCE } \\
\text { sensor }\end{array}$ & By fluorescence \\
\hline \multirow{4}{*}{$\begin{array}{l}\text { Solution from LaVie plastics } \\
\text { bottle }\end{array}$} & 0 & Not found & Not found & - & - \\
\hline & 5.00 & $4.98 \pm 0.06$ & $4.88 \pm 0.07$ & 99.53 & 97.23 \\
\hline & 10.00 & $10.28 \pm 0.14$ & $10.23 \pm 0.09$ & 102.83 & 102.26 \\
\hline & 15.00 & $14.49 \pm 0.29$ & $14.89 \pm 0.33$ & 96.60 & 99.20 \\
\hline
\end{tabular}

The recovery in each case is also shown.

commercial product in Vietnam, were cut into small pieces and washed several times with double distilled water. After vacuum drying, $1.0 \mathrm{~g}$ of the plastic sample and $50 \mathrm{~mL}$ of purified water were added to the flask, sealed, and heated at $70^{\circ} \mathrm{C}$ for 48 hours. Finally, the mixture is filtered by a $45 \mu \mathrm{m}$ filter. The as-prepared solution was divided into two parts: one for fluorescence measurement and the second part for electrochemical measurement in PBS pH 7. DPV voltammograms are shown in Figure S6. The emission spectra of BPA appeared at a wavelength of $506 \mathrm{~nm}$ (Figure S6, (b)). Table 2 shows concentrations of BPA spiked in the treated solution using prepared AuNDs/CTAB/GCE and the calculated recoveries by both techniques.

The concentration determinations using AuNDs/CTAB/ GCE were accurate, with a recovery range of $96.60 \%-$ $102.82 \%$. These were in agreement with data obtained using a FluoroMax-4 spectrofluorometer. Therefore, the detection performance of AuNDs/CTAB/GCE was acceptable for this purpose.

\section{Conclusion}

In this paper, the GCE-supported $\mathrm{Au}$ nanodendrite and CTAB (denoted as AuNDs/CTAB/GCE) have been developed by simple one-step electrodeposition. The AuNDs/ $\mathrm{CTAB} / \mathrm{GCE}$ sensor was able to detect BPA in a large concentration range $(0.025-10 \mu \mathrm{m})$ with a relative detection limit of $22 \mathrm{~nm}$. Sensor-to-sensor reproducibility was estimated by measuring seven separate prepared AuNDs/ CTAB/GCE sensors and the obtained RSDs were only less than $5.0 \%$. Real samples extracted from plastic drinking bottles were also tested and the determined concentrations were in good recoveries (96.60\%-102.82\%) and comparable with other conventional methods. Furthermore, AuNDs/ 
CTAB/GCE should be employable as an electrochemical sensor for on-site water analysis with low cost and without the serious worry of environmental contamination.

\section{Data Availability}

The graphical abstract, structure of study compound, SEM images at a larger scale, CVs and DPVs of sensor's reproducibility, and real sample analysis data used to support the findings of this study are included within the supplementary information files.

\section{Conflicts of Interest}

The authors declare that they have no conflicts of interest.

\section{Acknowledgments}

This research was funded by the Institute of Chemistry (grant number VHH.2020.2.01) and the Vietnam Academy of Science and Technology (grant numbers: NCVCC06.10/ 20-20 and КНСВHН.01/19-21).

\section{Supplementary Materials}

Fig. S1:structure of the studied compound. Fig. S2: SEM images of AuNDs created on GCE without (a) and with CTAB layer (b). Fig. S3: CVs of AuNDs/CTAB/GCE in $5 \mathrm{mM} \mathrm{K}_{3} \mathrm{Fe}(\mathrm{CN})_{6} / \mathrm{K}_{4} \mathrm{Fe}(\mathrm{CN})_{6}+0.2 \mathrm{M}$ PBS at different scan rates and used for calculation of electroactive surface area (ECSA) Fig. S4: reproducibility of seven AuNDs/CTAB/ GCE sensors at $10 \mu \mathrm{m}$ BPA in PBS pH 7. Fig. S5: voltammograms of BPA $5 \mu \mathrm{m}$ on AuNDs/CTAB/GCE before and after adding interferents at concentrations 20 times higher than that of analyte BPA with $\mathrm{Cd}^{2+}, \mathrm{Pb}^{2+}$, and $\mathrm{Cu}^{2+}$ (a) and with 4-nitrophenol (b). Fig. S6: voltammograms of solution extracted from plastic drinking water bottle spiked BPA at different concentrations recorded on a AuNDs/CTAB/GCE sensor (a) and by the fluorescence method (b). (Supplementary Materials)

\section{References}

[1] S. H. Safe, "Endocrine disruptors and human health: is there a problem? an update," Environmental Health Perspectives, vol. 108, no. 6, pp. 487-493, 2000.

[2] F. S. Vom Saal and C. Hughes, "An extensive new literature concerning low-dose effects of bisphenol A shows the need for a new risk assessment," Environmental Health Perspectives, vol. 113, no. 8, pp. 926-933, 2005.

[3] E. M. Malone, C. T. Elliott, D. G. Kennedy, and L. Regan, "Rapid confirmatory method for the determination of sixteen synthetic growth promoters and bisphenol a in bovine milk using dispersive solid-phase extraction and liquid chromatography-tandem mass spectrometry," Journal of Chromatography B, vol. 878, no. 15-16, pp. 1077-1084, 2010.

[4] Y. Ji, J. Yin, Z. Xu et al., "Preparation of magnetic molecularly imprinted polymer for rapid determination of bisphenol A in environmental water and milk samples," Analytical and Bioanalytical Chemistry, vol. 395, no. 4, pp. 1125-1133, 2009.
[5] Y. Kim, J. B. Jeon, and J. Y. Chang, "CdSe quantum dotencapsulated molecularly imprinted mesoporous silica particles for fluorescent sensing of bisphenol A," Journal of Materials Chemistry, vol. 22, no. 45, pp. 24075-24080, 2012.

[6] W. Zhao, N. Sheng, R. Zhu et al., "Preparation of dummy template imprinted polymers at surface of silica microparticles for the selective extraction of trace bisphenol A from water samples," Journal of Hazardous Materials, vol. 179, no. 1-3, pp. 223-229, 2010.

[7] B. De Meulenaer, K. Baert, H. Lanckriet, V. Van Hoed, and A. Huyghebaert, "Development of an enzyme-linked immunosorbent assay for bisphenol A using chicken immunoglobulins," Journal of Agricultural and Food Chemistry, vol. 50, no. 19, pp. 5273-5282, 2002.

[8] C. M. A. Brett and A. M. Oliveira-Brett, "Electrochemical sensing in solution-origins, applications and future perspectives," Journal of Solid State Electrochemistry, vol. 15, no. 7-8, pp. 1487-1494, 2011.

[9] A. Thamilselvan, V. Rajagopal, and V. Suryanarayanan, "Highly sensitive and selective amperometric determination of BPA on carbon black/f-MWCNT composite modified GCE," Journal of Alloys and Compounds, vol. 786, pp. 698706, 2019.

[10] K.-J. Huang, Y.-J. Liu, Y.-M. Liu, and L.-L. Wang, "Molybdenum disulfide nanoflower-chitosan-Au nanoparticles composites based electrochemical sensing platform for bisphenol A determination," Journal of Hazardous Materials, vol. 276, pp. 207-215, 2014.

[11] N. B. Messaoud, C. Dridi, M. B. Ali, and C. M. A. Brett, "Electrochemical sensor based on multiwalled carbon nanotube andgold nanoparticle modified electrode for the sensitive detection of bisphenol A," Sensors and Actuators B, vol. 253, pp. 513-522, 2017.

[12] T. D. Vu, P. Khac Duy, H. T. Bui, S.-H. Han, and H. Chung, "Reduced graphene oxide-Nickel sulfide (NiS) composited on mechanical pencil lead as a versatile and cost-effective sensor for electrochemical measurements of bisphenol A and mercury (II)," Sensors and Actuators B: Chemical, vol. 281, pp. 320-325, 2019.

[13] P. Hu, X. Zhu, X. Luo, X. Hu, and L. Ji, "Cathodic electrodeposited Cu-BTC MOFs assembled from $\mathrm{Cu}(\mathrm{II})$ and trimesic acid for electrochemical determination of bisphenol A," Microchimica Acta, vol. 187, p. 145, 2020.

[14] C. Hou, W. Tang, C. Zhang, Y. Wang, and N. Zhu, "A novel and sensitive electrochemical sensor for bisphenol A determination based on carbon black supporting ferroferric oxide nanoparticles," Electrochimica Acta, vol. 144, pp. 324-331, 2014.

[15] Y. Li, H. Wang, B. Yan, and H. Zhang, "An electrochemical sensor for the determination of bisphenol A using glassy carbon electrode modified with reduced graphene oxide-silver/poly-l-lysine nanocomposites," Journal of Electroanalytical Chemistry, vol. 805, pp. 39-46, 2017.

[16] R. Shi, X. Yuan, A. Liu, M. Xu, and Z. Zhao, "Determination of bisphenol A in beverages by an electrochemical sensor based on $\mathrm{Rh}_{2} \mathrm{O}_{3} /$ reduced graphene oxide composites," Applied Sciences, vol. 8, no. 12, p. 2535, 2018.

[17] S. U. Karabiberoglu, "Sensitive voltammetric determination of bisphenol A based on a glassy carbon electrode modified with copper oxide-zinc oxide decorated on graphene oxide," Electroanalysis, vol. 30, pp. 91-102, 2018.

[18] K. Deng, X. Liu, C. Li, Z. Hou, and H. Huang, "A comparative study of different $\mathrm{Fe}_{3} \mathrm{O}_{4}$-functionalized carbon-based nanomaterials for the development of electrochemical sensors for bisphenol A," Analytical Methods, vol. 9, no. 37, p. 5509, 2017. 
[19] G. Bolat, Y. T. Yaman, and S. Abaci, "Highly sensitive electrochemical assay for Bisphenol A detection based on poly (CTAB)/MWCNTs modified pencil graphite electrodes," Sensors and Actuators B: Chemical, vol. 255, no. 1, pp. 140148, 2018.

[20] H. Fan, Y. Li, D. Wu et al., "Electrochemical bisphenol A sensor based on N-doped graphene sheets," Analytica Chimica Acta, vol. 711, pp. 24-28, 2012.

[21] P. Jing, X. Zhang, Z. Wu et al., "Electrochemical sensing of bisphenol A by graphene-1-butyl-3-methylimidazolium hexafluorophosphate modified electrode," Talanta, vol. 141, pp. 41-46, 2015.

[22] Y. Lin, K. Liu, C. Liu et al., "Electrochemical sensing of bisphenol A based on polyglutamic acid/amino-functionalised carbon nanotubes nanocomposite," Electrochimica Acta, vol. 133, pp. 492-500, 2014.

[23] R. Shi, J. Liang, Z. Zhao, A. Liu, and Y. Tian, "An electrochemical bisphenol A sensor based on one step electrochemical reduction of cuprous oxide wrapped graphene oxide nanoparticles modified electrode," Talanta, vol. 169, pp. 3743, 2017.

[24] B. Su, H. Shao, N. Li, X. Chen, Z. Cai, and X. Chen, "A sensitive bisphenol A voltammetric sensor relying on AuPd nanoparticles/graphene composites modified glassy carbon electrode," Talanta, vol. 166, pp. 126-132, 2017.

[25] J.-Y. Wang, Y.-L. Su, B.-H. Wu, and S.-H. Cheng, "Reusable electrochemical sensor for bisphenol A based on ionic liquid functionalized conducting polymer platform," Talanta, vol. 147, pp. 103-110, 2016.

[26] T. Yang, H. Chen, R. Yang, Y. Jiang, W. Li, and K. Jiao, “A glassy carbon electrode modified with a nanocomposite consisting of molybdenum disulfide intercalated into selfdoped polyaniline for the detection of bisphenol A," Microchimica Acta, vol. 182, no. 15-16, pp. 2623-2628, 2015.

[27] T. Zhan, Y. Song, Z. Tan, and W. Hou, "Electrochemical bisphenol A sensor based on exfoliated Ni2Al-layered double hydroxide nanosheets modified electrode," Sensors and Actuators B: Chemical, vol. 238, pp. 962-971, 2017.

[28] J. Zhang, X. Xu, and L. Chen, "An ultrasensitive electrochemical bisphenol A sensor based on hierarchical Ce-metalorganic framework modified with cetyltrimethylammonium bromide," Sensors and Actuators B: Chemical, vol. 261, no. 15, pp. 425-433, 2018.

[29] H. Yin, Y. Zhou, S. Ai, R. Han, T. Tang, and L. Zhu, "Electrochemical behavior of bisphenol A at glassy carbon electrode modified with gold nanoparticles, silk fibroin, and PAMAM dendrimers," Microchimica Acta, vol. 170, no. 1-2, pp. 99-105, 2010.

[30] Y. Gao, Y. Cao, D. Yang, X. Luo, Y. Tang, and H. Li, "Sensitivity and selectivity determination of bisphenol A using SWCNT-CD conjugate modified glassy carbon electrode," Journal of Hazardous Materials, vol. 199-200, pp. 111-118, 2012.

[31] L. Zhang, Y.-P. Wen, Y.-Y. Yao, Z.-F. Wang, X.-M. Duan, and J.-K. Xu, "Electrochemical sensor based on f-SWCNT and carboxylic group functionalized PEDOT for the sensitive determination of bisphenol A," Chinese Chemical Letters, vol. 25, no. 4, pp. 517-522, 2014.

[32] H. Li, W. Wang, Q. Lv, G. Xi, H. Bai, and Q. Zhang, "Disposable paper-based electrochemical sensor based on stacked gold nanoparticles supported carbon nanotubes for the determination of bisphenol A," Electrochemistry Communications, vol. 68, pp. 104-107, 2016.
[33] X. Tu, L. Yan, X. Luo, S. Luo, and Q. Xie, "Electroanalysis of bisphenol A atmultiwalled carbon nanotubes-gold nanoparticles modified glassy carbonelectrode," Electroanalysis, vol. 21, pp. 2491-2494, 2009.

[34] C. $\mathrm{Hu}$ and $\mathrm{S}$. Hu, "Electrochemical characterization of cetyltrimethyl ammonium bromide modified carbon paste electrode and the application in the immobilization of DNA," Electrochimica Acta, vol. 49, no. 3, pp. 405-412, 2004.

[35] S. Hu, K. Wu, H. Yi, and D. Cui, "Voltammetric behavior and determination of estrogens at nafion-modified glassy carbon electrode in the presence of cetyltrimethylammonium bromide," Analytica Chimica Acta, vol. 464, no. 2, pp. 209-216, 2002.

[36] J. Gao and J. F. Rusling, "Electron transfer and electrochemical catalysis using cobalt-reconstituted myoglobin in a surfactant film," Journal of Electroanalytical Chemistry, vol. 449, no. 1-2, pp. 1-4, 1998.

[37] K. D. Pham, T. H. Y. Pham, S. Chun, T. T. H. Vu, and H. Chung, "Carbon fiber cloth-supported Au nanodendrites as a ruggedsurface-enhanced Raman scattering substrate and electrochemicalsensing platform," Sensors and Actuators B: Chemical, vol. 225, pp. 377-383, 2016.

[38] H. D. Vu, T. H. Y. Pham, Q. G. Nguyen et al., "Carbon fiber cloth-supported $\mathrm{Au}$ nanodendrites as a rugged surface-enhanced Raman scattering substrate and electrochemicalsensing platform," Electroanalysis, vol. 30, pp. 2222-2227, 2018.

[39] Q. G. Nguyen, H. D. Vu, T. H. Y. Pham et al., "Au nanodendrite incorporated graphite pencil lead as a sensitive, and simple electrochemical sensor for simultaneous detection of $\mathrm{Pb}(\mathrm{II}), \mathrm{Cu}(\mathrm{II})$ and $\mathrm{Hg}(\mathrm{II})$," Journal of Applied Electrochemistry, vol. 49, pp. 839-846, 2019.

[40] E. Laviron, "Adsorption, autoinhibition and autocatalysis in polarography and in linear potential sweep voltammetry," Journal of Electroanalytical Chemistry and Interfacial Electrochemistry, vol. 52, no. 3, pp. 355-393, 1974.

[41] S. Dong, G. Suo, N. Li et al., "A simple strategy to fabricate high sensitive 2,4-dichlorophenol electrochemical sensor based on metal organic framework Cu3(BTC)2," Sensors and Actuators B: Chemical, vol. 222, pp. 972-979, 2016.

[42] Report of joint FAO/WHO expert meeting, toxicological and health aspects of bisphenol A, 2-5 November, 2010.

[43] C. Li, Y. Zhou, X. Zhu, B. Ye, and M. Xu, "Construction of a sensitive bisphenol A electrochemical sensor based on metalorganic framework/graphene composites," International Journal of Electrochemical Science, vol. 13, pp. 4855-4867, 2018. 